Canadian University Music Review

Revue de musique des universités canadiennes

\title{
La discographie de Claude Vivier
}

Johanne Rivest

Numéro 6, 1985

URI : https://id.erudit.org/iderudit/1014031ar

DOI : https://doi.org/10.7202/1014031ar

Aller au sommaire du numéro

\section{Éditeur(s)}

Canadian University Music Society / Société de musique des universités

canadiennes

\section{ISSN}

0710-0353 (imprimé)

2291-2436 (numérique)

Découvrir la revue

\section{Citer ce document}

Rivest, J. (1985). La discographie de Claude Vivier. Canadian University Music Review / Revue de musique des universités canadiennes, (6), 35-44.

https://doi.org/10.7202/1014031ar

(c) Canadian University Music Society / Société de musique des universités canadiennes, 1985
Ce document est protégé par la loi sur le droit d'auteur. L'utilisation des services d'Érudit (y compris la reproduction) est assujettie à sa politique d'utilisation que vous pouvez consulter en ligne.

https://apropos.erudit.org/fr/usagers/politique-dutilisation/ 


\section{LA DISCOGRAPHIE DE CLAUDE VIVIER \\ Johanne Rivest}

PROLIF́RATION (1969, rév. 1975) 14'40"

Jean Laurendeau, ondes Martenot

Louis-Philippe Pelletier, piano

Serge Laflamme, percussion

Radio Canada International, RCI 358, 1973, épuisé

LETTURA DI DANTE (1974) 26'

Pauline Vaillancourt, soprano

Ensemble de la Société de musique contemporaine du Québec direction: Serge Garant

Radio Canada International, RCI 411, 1975

CHANTS (1972-73) 22'

Atelier de musique contemporaine de la Faculté de Musique,

Université de Montréal

direction: Lorraine Vaillancourt

Nocturnales, CCL-33-131, enregistré en 1978, produit en 1981

PULAU DEWATA (1977) 11'

Ensemble de percussion McGill

direction: Pierre Béluse

Radio Canada International, RCI 478, 1978

PIÈCE POUR FLÛTE ET PIANO (1975) 5'

Lise Daoust, flûte

Suzanne Blondin, piano

Radio Canada International, RCI 535, 1982

PULAU DEWATA (1977) 13'50"

ARRAYMUSIC: Doublas Perry, alto

Henry Kucharzyk, piano

Beverly Johnston, percussion 
SHIRAZ (1977) 12'

Louis-Philippe Pelletier, piano

LONELY CHILD (1980) 19'05"

Marie-Danielle Parent, soprano

Orchestre Métropolitain du grand Montréal

direction: Serge Garant

Centredisques, numérique, CMC 1384, 1984.

PULAU DEWATA (1977) 11'20"

Le Quatuor de Saxophones de Montréal:

Simon Stone, soprano

René Masino, alto

Dave Clark, ténor

Walter Boudreau, baryton

Société Nouvelle d'Enregistrement, SNE 515, 1985.

La discographie complète de Claude Vivier offre un aperçu assez global de sa production: de Prolifération, l'oeuvre qui l'a fait connaître en 1969, à Lonely Child, créée en 1980 et qui marque en aboutissement des tendances esquissées par Chants et Lettura di Dante - toutes deux composées lors du stage d'étude avec Stockhausen à Cologne (1972-74) - en passant par deux oeuvres inspirées de son voyage au Moyen-Orient, Pulau Dewata et Shiraz.

Les préoccupations vocales de Vivier sont clairement annoncées dans Prolifération, et ne cesseront de s'affirmer par la suite: sur disques, citons d'abord Chants, Lettura di Dante et surtout Lonely Child; dans le reste de sa production, des oeuvres tout autant significatives restent à signaler: Liebesgedichte (1976), Journal (1977), Love Songs (1977), l'opéra Kopernikus (1980) et sa dernière oeuvre, inachevée, Crois-tu en l'immortalité de l'âme ? (1983).

Une autre préoccupation importante de Vivier, la mélodie, sur laquelle se greffe des agrégats sonores que l'on perçoit surtout comme couleur, l'amène plus tard au concept de 'mélodie de couleurs de timbre', ou Klangfarbenmelodie. Cette dernière préoccupation s'amorce dans Lettura di Dante, mais n'aboutira significativement que dans Lonely Child. Les oeuvres strictement instrumentales procéderont de la même orientation, Pulau Dewata entre autres.

Quant à la puissance évocatrice de cette musique, elle est surtout dûe au traitement "allongé " du temps musical: sons soutenus, silences très longs, périodicité d'événements et forme en progression constante. 
Prolifération, gravé en 1973 par RCI, a été créée en 1969 alors que Vivier étudiait avec Gilles Tremblay, au Conservatoire de musique de Montréal. L'oeuvre, d'abord instrumentale, se mue graduellement en discours parlé: quelques mots surgissent, épars, de la trame musicale; petit à petit, ils se constituent en phrases et finissent par absorber complètement ce qui était musique.

Les trois interprètes de ce disque, impeccables sur le plan musical, semblent souffrir d'un manque notable d'imagination en ce qui a trait à l'improvisation verbale. Le final, en particulier, donne à la pièce un ton burlesque qui ne semble pas lui appartenir:

«- Bon ben, moé, j’ai fini.

- Le point final.

- Nous allons... écouter!"

L'effet transcendant, s'il devait en avoir un, se trouve ainsi anéanti. À voir le soulagement qu'éprouvent ces musiciens à clore leur tâche verbale, on se sent gêné: ici se trouve confirmé le préjugé qui confère au musicien professionnel un rôle timide et effacé, et pour qui l'extériorisation n'est possible qu'au moyen de son instrument. Â présent que le théâtre musical connaît plus d'adeptes, il conviendrait d'entreprendre une relecture de cette oeuvre, en partie aléatoire, afin que la musique de Vivier - laquelle se distingue de l'homme-Vivier - retrouve son climat de religiosité inspirée.

Lettura di Dante est écrite pour voix de soprano et orchestre de chambre. Le soprano Pauline Vaillancourt et la Société de Musique Contemporaine du Québec en donnent une interprétation magistrale. On connaît bien la qualité des exécutions du directeur de cette société dans les milieux musicaux montréalais, lequel a créé de nombreuses oeuvres de jeunes compositeurs dont Lettura di Dante et Liebesgedichte de Claude Vivier, avec la participation de Pauline Vaillancourt.

Lettura di Dante exploite ce thème, cher à Vivier, de l'éternité. Une " mélodie qui revient toujours et qui se transforme en même temps " ${ }^{1}$, une chanteuse qu'on ne voit pas, sauf lorsqu'elle se met à réciter quinze fois de suite " Ho visto dio " (j'ai vu Dieu), bref une oeuvre qui s'élabore en spirale, nous amenant « de l'enfer au ciel "2, à la manière de la Divine Comédie de Dante. Les textes de Claude Vivier, chantés en italien, utilisent cette langue simplement à cause de sa musicalité.

Par cette musique intense, d'apparence ultra-sensible plutôt que cérébrale, Vivier désirait se démarquer de l'ensemble de la 
production contemporaine occidentale. Il recherchait l'accès à une nouvelle sensibilité, plus proche de l'individu:

La musique que je fais (...) décèle certaines douceurs ou certaines associations avec l'enfance, elle réveille certains instincts de tendresse, de bonté, d'amour. La musique occidentale savante a perdu une qualité de naturel, une musicalité naturelle qui n'est pas arrangée. C'est cette musicalité naturelle que j'essaie de retrouver. ${ }^{3}$

C'est au moment où Vivier arrive à Cologne, en 1972, qu'il éprouve cette nécessité de trouver sa vraie voie. Chants se définit comme une musique à processus, laquelle repose sur " l'enchaînement de situations musicales, dramatiques, textuelles ou autres " . ${ }^{4}$ La situation musicale s'exprime par une cellule mélodique basée sur un mode de Messiaen; huit sections décrivent textuellement le parcours de l'oeuvre; quant à la situation dramatique, elle est ainsi exposée par Vivier: "L'oeuvre entière est un rite de la mort et de la naissance à la nouvelle lumière, celle de l'enfance et de toute sa pureté, aussi la lumière de la liberté face à la mort $"{ }^{5}$.

Sept voix de femme - trois voix principales, trois voix secondaires - dont la septième est une sorte d'alter-ego du compositeur, évoluent a capella, tantôt en français, en latin, ou en langue inventée, tantôt parlant, tantôt chantant. Sur un fond sonore à texture plutôt homophonique, une ou plusieurs voix se découpent. C'est le cas, notamment, de la septième voix qui débute de façon impromptue, avec un timbre vraiment particulier exposant une longue phrase parlée, laquelle est répétée en écourtant la fin à chaque fois, jusqu'à ce qu'elle disparaisse tout à fait.

Cette inclusion du sujet dans l'oeuvre, sa visibilité, permet un détachement, un discours du « je " sur le « je ". Ainsi Vivier donne à voir non seulement son individualité propre, mais l'interprétation de cette individualité, sorte de dissertation sur lui-même. C'est pourquoi il est mal à-propos d'attribuer à Vivier l'épithète de "romantique ": poser le sujet dans l'oeuvre d'art est résolument moderne. ${ }^{6}$

L'interprétation de l'Atelier de musique contemporaine, sous la direction de Lorraine Vaillancourt, fait très bien ressortir la personnalité des voix. Les ponctuations occasionnelles de percussion ont été cependant mal enregistrées, ce qui occasionne d'agaçantes distorsions. Ce détail ne réussit cependant pas à 
détruire la beauté sensible qui se dégage de l'oeuvre, car les voix possèdent une force de conviction particulière.

La recherche d'une " nouvelle musicalité » surgit et renvoie à l'intérêt grandissant des compositeurs de la jeune génération pour la musique ethnique et son incorporation - pour certaines de ses particularités esthétiques ou formelles - dans leurs créations.

Un séjour à Bali, en 1976, inspire à Vivier Pulau Dewata, littéralement, " île des dieux ». L'oeuvre, composée pour n'importe quelle combinaison d'instruments dans une écriture à quatre voix, a fait l'objet de trois enregistrements. Le plus ancien, par l'ensemble torontois Arraymusic, a été enregistré sept ans avant sa parution en 1984 sur le disque consacré à Vivier (CMC 1384). Il s'agit d'un arrangement pour alto, piano et percussion (marimba, carillon et tam-tam), exécuté sous la direction de Claude Vivier en 1977, et d'une durée totale de 13'50.

La seconde version a été effectuée par l'Ensemble de percussion McGill de Montréal, dédicataire de l'oeuvre, en 1978 (RCI 478). L'Ensemble, dirigé par Pierre Béluse, adapte l'oeuvre à quatre marimbas, dont quelques passages confiés aux cloches tubulaires et au glockenspiel. L'exécution, de 11 minutes, est la plus brève des trois.

La version la plus récente, de 1985, propose une autre variante de cette oeuvre caméléon : le Quatuor de Saxophones de Montréal, qui avait été salué à deux reprises en 1983 - d'abord en novembre, à l'occasion d'un concert de la Société de Musique Contemporaine du Québec, puis en juin, lors du concert commémoratif à la mémoire du compositeur - a repensé l'oeuvre en fonction d'une articulation antiphonale de la mélodie et de la perception des harmoniques par l'élimination du vibrato.

Les trois réalisations n'offrent en définitive que peu de similitudes, chacune utilisant des procédés d'écriture, d'instrumentation ou d'interprétation fort divergents.

Arraymusic, par l'instrumentation hétérogène et plus traditionnaliste (piano - alto), permet plus de lyrisme à saveur romantique que les deux autres versions. Connaissant les tendances spirituelles éthérées du compositeur, lequel, ne l'oublions pas, dirige l'ensemble, une telle emphase sur l'expressivité ne surprend guère : au contraire, il nous semble ainsi assister à la révélation de ce respectueux "hommage d'amour pour ce merveilleux peuple qui m'a tant appris " 7 , selon les mots mêmes de l'auteur. Â certains moments, la couleur du timbre de chaque instrument arrive à créer une teinte d'exotisme: le marimba 
surtout, avec ses tremolandi pour soutenir les valeurs longues, rappelle l'orchestre balinais, comme parvient à le faire également le piano en répétant percussivement des grappes d'accords. L'alto, lorsqu'il se décide à abandonner son vibrato inspiré, prend l'allure nasillarde et frêle du rabâb des gamelans.

Sur le plan de l'effet exotique, la version à quatre marimbas de l'Ensemble McGill est cependant plus immédiate. Homogénéité des timbres, certes, mais recherche de contrastes par des oppositions de nuances et par l'introduction occasionnelle des cloches tubulaires ou du glockenspiel. Comparé à la version d'Array music, celle-ci perd en lyrisme ce qu'elle gagne en clarté. Le martèlement incessant d'un petit nombre d'instruments, sans mise en relief possible des courts motifs (car la dynamique des marimbas, manquant de souplesse, opère par blocs), ressemble alors à une application de solfège métrique. Aucun artifice d'exécution, ni rallentendo, ni decrescendo (sauf aux cloches tubulaires, dans un passage transitoire), ne vient en diversifier significativement l'effet, ne réussit à provoquer l'éveil de stimuli variés chez l'auditeur. Il s'ensuit une impression quelque peu désagréable de monotonie, de froideur, ou encore de superficialité juvénile, ceci renforcé par le caractère dansant qu'une telle exécution affirme.

Le Quatuor de Saxophones, quant à lui, réussit l'amalgame de l'expression et de la simplicité proprement balinaise : la grande souplesse dynamique de ces instruments alliée au caractère distinctif de leurs timbres selon les registres, l'arrangement de Walter Boudreau (saxophoniste baryton de l'ensemble, aussi compositeur et chef d'orchestre) étudié afin de faire ressortir la structure compositionnelle, bref tout concourt ici à nous faire apercevoir ce qui doit être l'intention de l'auteur. L'exclusion du vibrato permet une fusion des sons, laquelle génère une mélodie résultante, elle-même formée des sons résultants ou harmoniques.

Les sections ne sont plus simplement découpées en zones larges comme chez l'Ensemble de percussion, mais en plusieurs niveaux, ce qui donne un éclairage neuf concernant la structure : plus qu'une charpente élémentaire, l'arrangement éclaire une structure complexe où, par l'alternance des timbres et des registres en relation avec la texture mélodique ou homophonique de la composition, il devient possible de repérer un jeu d'accents et de désinences, à des distances temporelles variables, provoquant un ciselage dans l'ensemble. Comme chez Array music, on retrouve un certain sens dramatique : les silences prolongés sont significatifs, 
chargés d'émotion. La fin en est cependant moins lyrique, plus sobre, sans recherche d'effets. La pulsation égale, sans rallentendo, n'a pas le caractère maladroit de l'Ensemble McGill, car là où les marimbas, sans finesse, exécutent telle quelle la notation, le Quatuor articule les notes de façon à créer des contours affirmés. "La fin de la pièce, dit le compositeur, est la signature traditionnelle de nombreuses oeuvre balinaises " ${ }^{8}$, aspect dont Arraymusic ne tient certainement pas compte, avec sa finale méditative, proche du silence, où le calme renvoie à une aspiration spirituelle élevée, qui laisse à son tour deviner la mort dans toute sa vénérabilité.

Le microsillon de 1982 consacré à un ensemble composite d'oeuvres pour la flûte traversière moderne, comporte la courte Pièce pour flûte et piano, composé en 1975 pour servir de morceau de concours au "tremplin international" des Concours de musique du Canada. Selon le compositeur, il s'agit d'un amalgame de différents extraits d'oeuvres sur lesquelles il travaillait à l'époque. Cette pièce progresse du grave vers l'aigu, de " piano " à "forte ", alant du style méditatif au tumulte grandissant. L'arrêt final se fait brusque, après une telle intensification du matériau.

Ce matériau, on en reconnaît l'empreinte : alternance de moments calmes et soutenus avec l'effervescence de traits rapides, entrecoupés d'arrêts pour reprendre haleine. Les procédés d'écriture qui s'y trouvent sont une sorte de prémonition de Shiraz qui les exploitera beaucoup plus à fond. Les deux interprètes de cette oeuvre sont issues du Conservatoire de musique de Montréal, mais ont ensuite travaillé à l'étranger. L'interprétation qu'elles défendent est satisfaisante, bien que le vibrato de Lise Daoust gagnerait à être mieux contrôlé dans les longues tenues.

Shiraz tire également son inspiration d'ailleurs : l'Iran, plus précisément la ville de Shiraz, laquelle, par l'aspect ciselé de son paysage, amène Vivier à " tailler " l'oeuvre par des mouvements des mains sur le piano - instrument soliste de l'oeuvre. L'écriture, homophonique, comporte quatre voix, deux par main; au centre émerge un contrepoint à deux voix, puis l'oeuvre se termine par un choral. Les mouvements des mains s'effectuent de trois façons principales - stables, parallèles ou opposés - et de leurs permutations. Tous les paramètres se déroulent de façon discontinue par rapport à un axe horizontal; ils sont morcelés en sections et sous-sections, ce qui crée des vides, des attentes, des indécisions mais qui, en définitive, facilite les changements de direction, permet une réorientation constante, agit semblablement 
au phénomène de la réfraction. Rien de très prévisible dans cette pièce au profil on ne peut plus escarpé. Après tous ces faux départs, ces fausses arrivées, ces moments creux ou en surplomb, ces retours brusques, sans transition, on ressent comme un malaise, celui d'un immense statisme, un enfermement à l'intérieur des limites mêmes du clavier. Le dynamisme des courtes sections mélodiques et rythmiques s'élabore en pure perte, à cause, d'une part, des répétitions incessantes qui réfrènent l'élan, et, d'autre part, de la discontinuité de la mélodie, laquelle perd ainsi tout sens de direction. Notre horizon est constamment fractionné par des éléments qui contiennent leurs propres points de rupture, et qui génèrent des unités qui s'entrechoquent.

Ma musique est un paradoxe. Habituellement, en musique, nous avons un développement, une direction ou un but (...). Je n'ai que des énoncés musicaux qui, de toute manière, ne conduisent nulle part. Pourtant, ils mènent aussi quelque part, mais cela repose sur une base beaucoup plus subtile. ${ }^{9}$

Cette utilisation du matériau s'inscrit donc dans la " manière " de Claude Vivier et ne concerne pas plus Shiraz qu'une autre oeuvre. Ce refus de la continuité de la ligne mélodique l'incite à fouiller davantage le son, à en élargir le développement interne. C'est ce qui se produit avec Lonely Child, assurément la plus significative des oeuvres qui composent cette discographie.

L'oeuvre est écrite pour voix de soprano et orchestre ; dédiée à Louise André, professeur de chant montréalais, elle a cependant été écrite à l'intention de Marie-Danielle Parent, élève de la précédente, qui l'a créée en 1980 et qui est la soliste du présent enregistrement. Lonely Child, c'est un hommage à l'enfance, à la pureté et à l'immortalité qu'elle incarne. L'enfant, rédempteur de l'existence terrestre, maintient en éveil le rêve et l'imaginaire. La spiritualité de cet être tangible conduit à l'intangible, l'éternité. Le texte de Vivier, très suggestif, a été composé postérieurement à la musique. Il utilise tour à tour le français et une langue inventé, "a-sémantique, dont la valeur ne réside que dans les sons eux-mêmes ". 10

L'oeuvre, malgré la constance de sa progression, se déroule en quatre sections principales, séparées par des coups de grosse caisse : un seul entre les sections 1 et 2,2 et 3 , dix plus deux coups aux cloches tubulaires entre les $3^{\mathrm{e}}$ et $4^{\mathrm{e}}$ sections. Les première et dernière sections sont en écriture homophonique, c'est-à-dire que c'est la mélodie qui prédomine et que chacune des notes se trouve 
" colorée " par des mixtures de timbres et de hauteurs. Les deux sections centrales sont plutôt polyphoniques, c'est-à-dire que l'homophonie initiale est dissoute par une sorte de désordre rythmique et harmonique, ce qui donne une impression de chaos. Les passages homophoniques sont ponctués par des coups de ching, ce qui rappelle certaines musiques orientales, du Japon entre autres. L'oeuvre évolue grosso modo du calme - chanté en français - vers une effervescence anxieuse - langue inventée -, puis retourne graduellement au calme, par soubresauts - français, langue inventée - pour atteindre à nouveau la sérénité du début -français. Plus encore qu'un climat, c'est la mélodie du début que l'on retrouve textuellement à la fin. Une forme close correspondant au mouvement même de la vie, où naissance et mort se rejoignent. L'enfance symbolise alors le commencement et la fin : état hors duquel on est projeté dans la vie courante, état de bienfaisance vers lequel on aspire au terme de son existence. Quelques mois précédant sa mort, Vivier écrit :

Comme c'est étrange. Malgré tout ce que je t'ai dit sur l'adulte que j'étais devenu la seule voix qui perce en moi, c'est celle de l'enfant qui parle doucement aux anges le soir! Tout autour de moi c'est le silence. ${ }^{11}$

Malgré son originalité, la musique de Claude Vivier s'inscrit dans un courant dont le chef de file est d'abord ce compositeur italien redécouvert en France dans la dernière décade, Giacinto Scelsi (né en 1905). La musique de ce dernier ${ }^{12}$ est dépouillée de tous les paramètres habituels pour ne concentrer que sur les couleurs, engendrées par une série d'harmoniques superposées sur un son fondamental, avec un ensemble hétérogène d'instruments où les sons instables - fluctuations de micro-intervalles - engendrent à leur tour des frottements, ce qui épaissit la texture musicale. Les notes ou les quelques intervalles se répètent inlassablement, à un rythme régulier et large. Les sonorités ainsi produites rappellent les musiques incantatoires du Japon ou de la Corée. Les compositeurs français issus de cette pensée, appartenant à ce nouveau courant appelé " néo-romantisme ", ont, comme figures de proue, Gérard Grisey et Tristan Murail. ${ }^{13}$ Le premier, dans son traité non publié Tempus ex Machina, réflexions sur le temps musical, propose une application de principes électro-acoustiques à la musique instrumentale - où la " profondeur " du son est exploitée - et un retour à certaines lois de la perception physiologique - par exemple, de renouer avec la périodicité. 
La musique de Claude Vivier est-elle géniale ? Ce qui importe, c'est qu'elle ait quelque chose de particulier à dire, et qu'elle le prononce d'une façon qui nous touche actuellement, avec sa voix si personnelle, malgré tout, et si attachante. Quant à la discographie, elle reste à poursuivre avec des oeuvres comme Orion, Kopernikus... Présentement, le meilleur disque à acquérir est certainement l'avant-dernier, où la prise de son est nettement supérieure, en plus d'offrir l'intérêt de présenter trois oeuvres importantes du compositeur, interprétées avec la ferveur de l'hommage au disparu.

\section{NOTES}

1. "Vivier à Claude Gingras ", La Presse (Montréal), 24.09.74.

2. "Vivier à Jacques Thériault", Le Devoir (Montréal), 20.09.74.

3. "Vivier à D. Carrière ", Le Berdache No. 22 (Montréal), juilletaoût 81.

4. Vivier, Notes de pochette du disque CCL-33-131.

5. Vivier, Texte de présentation de Chants au Centre de musique canadienne.

6. À ne pas confondre avec le "nouveau romantisme", qui est un concept se rapportant à la démarche des compositeurs des années 70-80, où la manie de structuration du discours musical se voit remplacée par la souplesse d'un procédé plus intuitif et relié à la perception psychologique plutôt qu'au pur plaisir cérébral. Vivier appartient au courant de cette décade.

7. Vivier, notes de pochette des disques RCI 478 et CMC 1384.

8. Ibid.

9. "My music is a paradox. Usually in music you have some development, some direction or some aim (...). I just have statements, musical statements, which somehow lead nowhere. Also on the other hand, they lead somewhere, but it's on a much more subtle basis. "Vivier, "Interview with Susan ", Musicworks, No. 18, hiver 1982.

10. Vivier, Notes de pochette du disque CMC 1384.

11. Vivier, Lettre à une amie (26.01.83), citée in Programme-souvenir du Concert à la mémoire de Claude Vivier, Montréal, le 2 juin 1983.

12. Par exemple, Quatro Pezzi (quatre pièces sur une seule note), disponible sur FY103, RC 350.

13. Selon Anne LeBaron et Denys Bouliane, "Darmstadt 1980 ", Perspectives of New Music, XIX (1980-81), pp. 420-41. 